\title{
Effect of surface temperature on tribological behavior of polytetrafluoroethylene composites
}

\author{
M. Conte ${ }^{1,2}$, B. Fernandez $z^{3}$ \& A. Igartua ${ }^{3}$ \\ ${ }^{1}$ Anton Paar TriTec SA, Switzerland \\ ${ }^{2}$ Empa, Laboratory for Mechanics of Materials and Nanostructures, \\ Switzerland \\ ${ }^{3}$ IK4-Tekniker, Spain
}

\begin{abstract}
The tribological behavior of polytetrafluoroethylene is highly affected by fillers used to improve its mechanical characteristics and depends on the ability of the material to maintain its integrity under working conditions. Specifically, in the case under study, the heat generated by friction was modelled as a heat flow coming from a source at the contact surface in static condition. Considering that a heat flow is also supplied to the samples during a differential scanning calorimetry analysis, when the crystallinity of the material is evaluated, a correlation between the thermal characteristics of the material, its structure and tribological behavior is presented.
\end{abstract}

Keywords: PTFE, friction, heating, wear, temperature rise.

\section{Introduction}

Polytetrafluoroethylene (PTFE) is well known for its low coefficient of friction in dry conditions even if it presents low carrying capacity, which is a reason why manufacturers have been trying to improve its mechanical characteristics by means of suitable fillers building a wide family of PTFE composites [1]. The results have been quite interesting but, in the end, it has been seen that such fillers compromise PTFE's attractive low friction properties $(\mu<0.1)$ even if wear of such composites in comparison with plain PTFE is further reduced [2]. Furthermore, a disadvantage of using hard phases in soft matrix is well known, namely, the possibility of transfer film removal and the aggressive abrasion of the hard counterface if the hard phases are released to the interface as a third body due to 
loosening of the bond between the filler and the polymer matrix: it was found that the particles used as fillers were rubbed off during the test, highlighting the weakness of the bond between the transfer film and the countersurface [3]. The role of the transfer film has also been extensively investigated, but not too much importance has been given to the role of temperature at the contact surfaces and its effect on the characteristics of PTFE composites, probably due to understandable difficulties in experimental measurements. Frictional heating calculations in fact are quite difficult and imperfect because of the lack of knowledge about many of the underlying assumptions $[4,5]$. Parameters such as normal load and sliding velocity affect the presence of thermal energy from frictional resistance but material properties and friction coefficient are constantly changing, and if it is consider that the latter enters the flash temperature computation to the first power, it is clear that its variation cannot be considered negligible, especially during the running-in period [6]. The aim of this work is the interpretation of the tribological behavior of PTFE and PTFE composites in relation to contact temperature and their thermal characteristics, as well as their structure.

\section{Experimental setup and specimens}

Friction and wear tests were carried out in the test rig represented in fig. 1: a PTFE sample (1) is mounted on a frame (2) and loaded by an axially sliding rod of CrVNiMo (3) supported and fixed by two holders (4) on which a normal constant load $W$ is applied and transmitted to the axis of the rod. The holders and a couple of screws prevent rotation of the rod around its axis. The system is located within a climate chamber in order to set temperature and humidity percentages. The normal load was set at $50 \mathrm{~N}$ for the first $30 \mathrm{~s}$ and then at $100 \mathrm{~N}, 60 \mathrm{~Hz}$ frequency and $2 \mathrm{~mm}$ stroke for each sample. The coefficient of friction $(\mathrm{CoF})$ was recorded at regime condition for 30 minutes; each test was repeated 2-3 times.
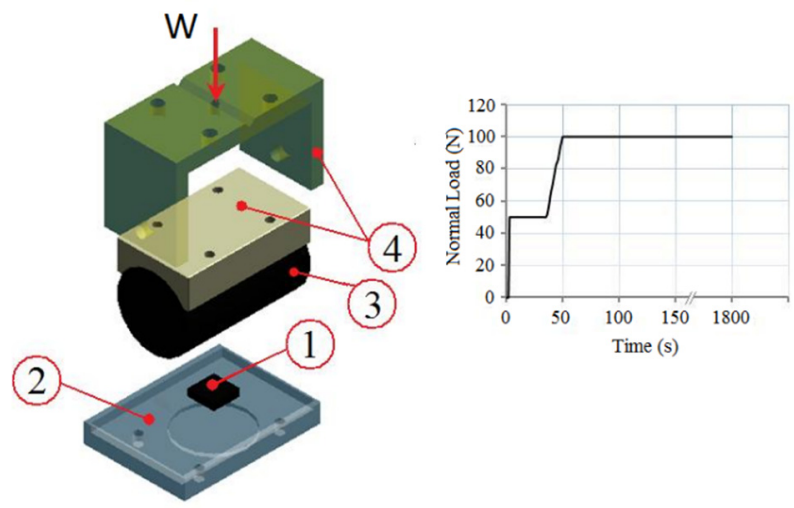

Figure 1: Test rig configuration: PTFE sample (1), frame (2), sliding rod (3), holders (4). Normal load is set at $50 \mathrm{~N}$ for the first $30 \mathrm{~s}$ and then at $100 \mathrm{~N}$. 
Taking the plain PTFE as reference, three commercial PTFE composites were tested in sliding contact against a CrVNiMo rod (table 1). According to the provider's communications, the PTFE samples come from a process of compression molding of the PTFE and filler powder mixtures, and subsequently sinterization and cooling. The PTFE dispersion has a median particle size of $0.25 \mu \mathrm{m}$; the average carbon particle diameter is about $10-25 \mu \mathrm{m}$; the average bronze particle size is about $25 \mu \mathrm{m}$; the glass fibers are about $5 \mu \mathrm{m}$ in diameter and $90-120 \mu \mathrm{m}$ in length.

Table 1: Thermal characteristics of the samples.

\begin{tabular}{|l|l|c|c|c|c|c|c|c|}
\hline & Sample & $\begin{array}{c}\text { Thermal } \\
\text { conductivity } K \\
(\mathrm{~W} / \mathrm{mK})\end{array}$ & $\begin{array}{c}\text { Thermal } \\
\text { diffusivity } \chi \\
\left(\mathrm{m}^{2} / \mathrm{s}\right)\end{array}$ & $\begin{array}{c}\text { Melting } \\
\text { temperature } \\
T_{m}\left({ }^{\circ} \mathrm{C}\right)\end{array}$ & $\begin{array}{c}\text { Heat of } \\
\text { fusion } \\
(\mathrm{J} / \mathrm{g})\end{array}$ & $\begin{array}{c}\Delta m \\
(\mathrm{mg})\end{array}$ & $\begin{array}{c}E \\
(\mathrm{MJ})\end{array}$ & $\begin{array}{c}E_{w} \\
(\mathrm{MJ} / \mathrm{g})\end{array}$ \\
\hline 1 & $\begin{array}{l}\text { PTFE } \\
\text { Virgin } \\
(\mathrm{REF})\end{array}$ & 0.24 & $9.24 \mathrm{e}^{-8}$ & 330 & 27.80 & 5.3 & 9.0 & 1.7 \\
\hline 2 & $\begin{array}{l}\text { PTFE } \\
+25 \mathrm{wt} \% \\
\text { Carbon }\end{array}$ & 0.45 & $2.04 \mathrm{e}^{-7}$ & 330 & 19.09 & 0.4 & 10.5 & 26.4 \\
\hline 3 & $\begin{array}{l}\text { PTFE } \\
+60 \mathrm{wt} \% \\
\text { Bronze }\end{array}$ & 0.46 & $1.86 \mathrm{e}^{-7}$ & 329 & 11.73 & 1.1 & 14.2 & 12.9 \\
\hline 4 & $\begin{array}{l}\text { PTFE } \\
+25 \mathrm{wt} \% \\
\text { Glass } \\
\text { fibers }\end{array}$ & 0.44 & $1.88 \mathrm{e}^{-7}$ & 331 & 18.91 & 0.7 & 9.9 & 14.2 \\
\hline
\end{tabular}

PTFE composites with carbon, bronze and glass fiber fillers were chosen in order to evaluate the effect of their presence and their different ways of acting.

Optical microscopy was used for investigating the worn surface. Differential scanning calorimetry (DSC) was used to study the thermal transition of polymers and to evaluate the crystallinity of the semi-crystalline materials. The dimension of the crystals depends on the mobility of the polymeric chains, easier at higher temperatures, and is influenced by the content of fillers and their nature. The thermal traces in fig. 2 can be interpreted by beginning with the concept of the heat of fusion: the ratio of the total heat input, that is, the area between the melting peak and the linear extrapolation of the curve after the glass transition, to the mass of the sample. This ratio represents the amount of heat absorbed per unit mass of the material during melting. The thermal traces in fig. 2 show no additional reaction between the filler materials and the matrix and no variation in the position of the transition-melting peak. On the other hand, the heat of fusion of the bulk PTFE polymer is significantly greater than that of the bulk composites. This indicates a higher thermal stability for the pure polymer even if great importance has to be given to the slope of the samples (1), (2) and (3) between the glass transition phase and the starting of the melting transition phase (approximately between 30 and $290^{\circ} \mathrm{C}$ ) and corresponding to a reduction of the supplied heat flow. After glass 
transition, in fact, polymers have high mobility increasing their temperature up to creating ordered dispositions called crystals and subsequently they release heat. Observing the behavior of the PTFE composite (4), it seems that glass fibers constrain the polymer chains more than carbon and bronze particles probably due to their bigger size and content in $\mathrm{wt} \%$, inducing a higher level of stability.

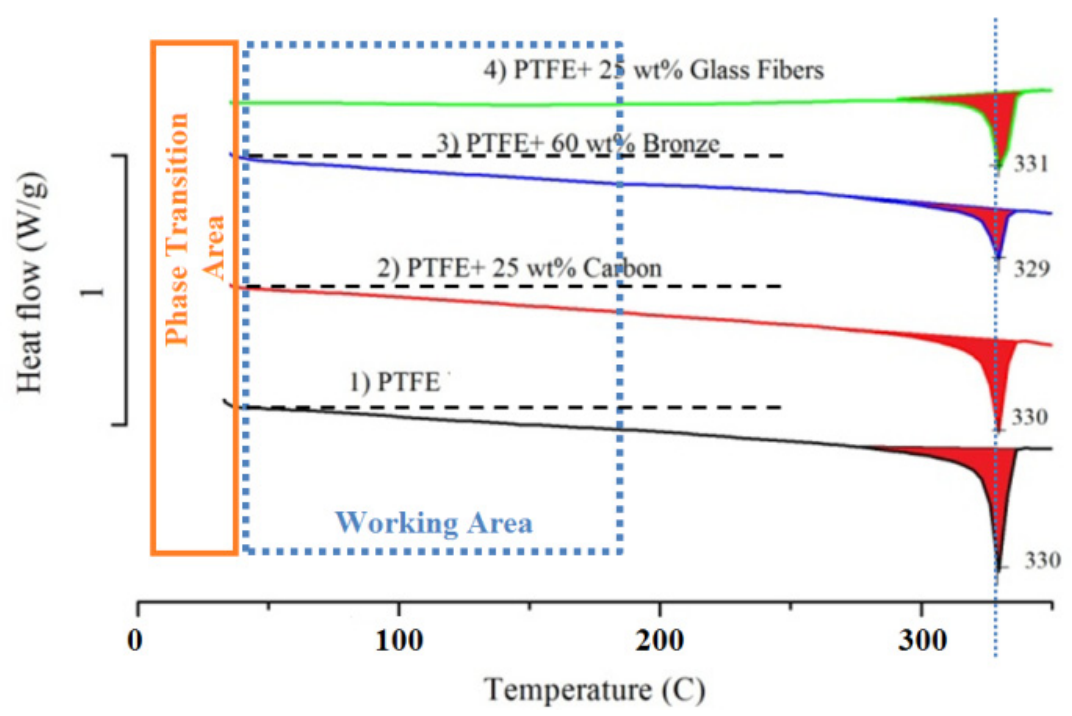

Figure 2: Thermal transition of PTFE samples and corresponding melting temperatures. Integration lines come from linear extrapolation from the end of the glass transition.

A better comprehension of the PTFE behaviour needs the understanding of the role played by its crystallinity, as already described by the authors in detail [7]. In fact, PTFE exhibits complex crystalline phase behavior: at atmospheric pressure, three crystalline structures (phase II, IV and I) are observed with transition between them occurring at 19 and $30^{\circ} \mathrm{C}$ (fig. 3), the first few working cycles in practical situations.

Brown and Dattelbaum [8] found that crack propagation in PTFE is strongly phase dependent with a brittle-to-ductile transition in the crack propagation behavior associated with the two room temperature phase transitions.

In [7], it was found that the presence of fillers does not affect the polymer melting point and the transition phase points but plays an important role in strengthening the bulk PTFE.

From a tribological point of view, the sliding contact between two materials results in heat generation at the asperities and consequently in increments of temperature at the interface. It means that the wear resistance of the material to adhesion depends upon the ability of the material to maintain its integrity at the resultant temperature due to the heat generated by friction and the way this heat is dissipated. Thus, filler material characteristics such as thermal conductivity and 


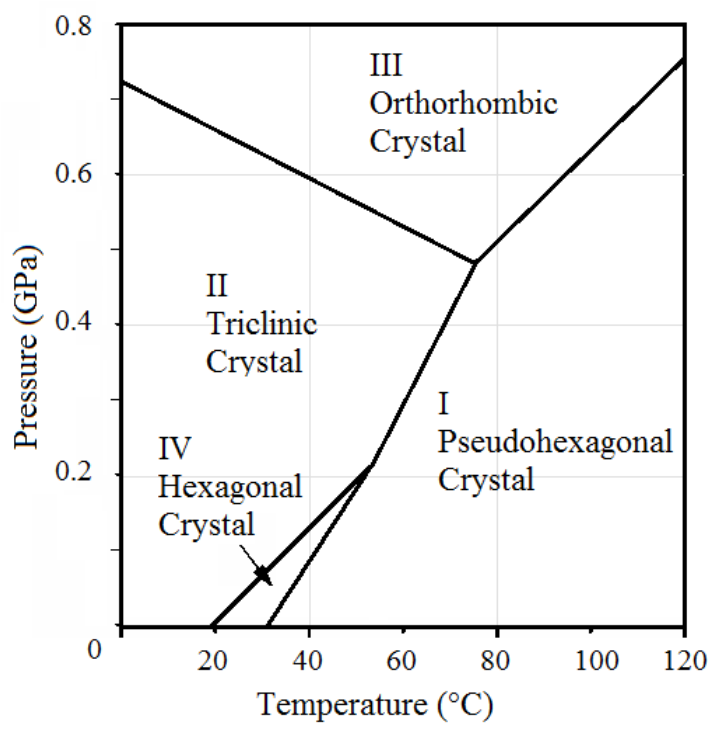

Figure 3: $\quad$ PTFE phase diagram at low pressure.

thermal diffusivity have a significant effect on the tribological properties of the PTFE composites.

This should not indicate that the only way to crystallize is due to temperature, but that these kinds of materials show great propensity to crystallize under stretched conditions whereas they crystallize slowly under rest conditions.

\section{Tribological tests and frictional heating calculations}

When a surface contact experiences frictional heating, an unsteady situation ensues because the temperature rise is a function of time and position. The transient behavior is determined by the geometry of the thermal source, the thermal properties of the materials and relative velocity. In the case under study, where the conductivity and diffusivity of the rod is much higher than those of the polymers, starting from the flash temperature rise calculation illustrated in [6], the following assumptions were adopted: a linear contact area as thermal source; a steady state conductive heating thanks to the short stroke of the sliding movement in comparison with the contact length. The latter assumption permits to avoid the calculation of the Peclet number [6], a parameter highly affected by the heat capacity variation of the polymer over temperature. Considering the work done by the friction force $Q$ over time as given by

$$
Q(t)=\mu(t) \cdot W \cdot v
$$

where $\mu$ is the coefficient of friction, $W$ the normal load and $v$ the sliding velocity, the contact temperature can then be described also as function of time by 


$$
T_{f \max }(t)=\frac{2 \cdot Q(t)}{K \cdot A} \cdot \sqrt{\frac{2 \cdot \chi \cdot b}{\pi \cdot v}}
$$

where $K$ is the thermal conductivity, $\chi$ the thermal diffusivity, $A$ the contact surface and $2 b$ the contact width. The contact area considered for calculations in the eqn. (2) is the cylindrical sector of the contact zone at the end of the test, which is not reliable to measure or to hypothesize its evolution over time: it means that the calculated temperature was under-estimated. In the case under study, the differences observed between the contact areas of the samples were so small that their variation did not affect the calculation and the value was approximately $9 \mathrm{~mm}^{2}$.

Eqns (1) and (2), thus, describe the progressive evolution of the work done by the friction force and the temperature evolution at the contact. In figs 4 to 6 , the coefficient of friction and the temperature are plotted over time: the curves are the same except from a multiplication factor depending on the geometrical dimensions of contact and the thermal characteristics of the materials. The importance of taking into account the effect of the fillers from the structural and thermal point of view becomes clear looking at the coefficient of friction of plain PTFE and its maximum contact temperature (fig. 4): if its thermal conductivity is half that of the PTFE composites, its thermal diffusivity is an order of magnitude lower delaying the cooling of the polymer and resulting in an increment of temperature.

Considering the thermal transition curves of PTFE composites in fig. 2 , it is possible to see how the composites numbered 2, 3 and 4 can be affected by the heat flux generated at the contact, as previously explained; this means that small crystals are formed in that range of temperature, that is, less stable crystals start the wear processes. The presence of high $\mathrm{wt} \%$ of bronze (composite 3 ) avoids the formation of bigger crystals (more stable) so the wear resistance results are significantly affected.

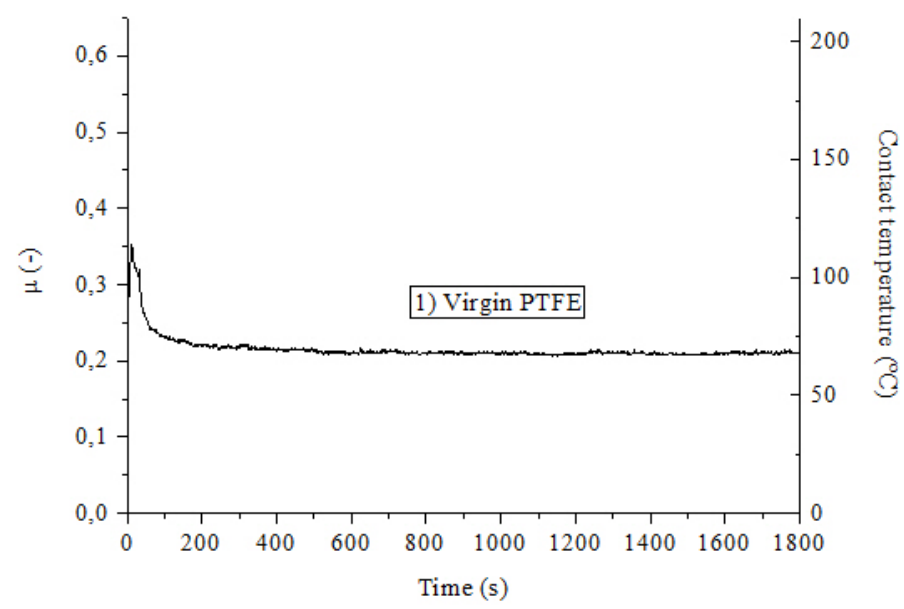

Figure 4: Coefficient of friction and calculated contact temperature evolution of plain PTFE sample. 


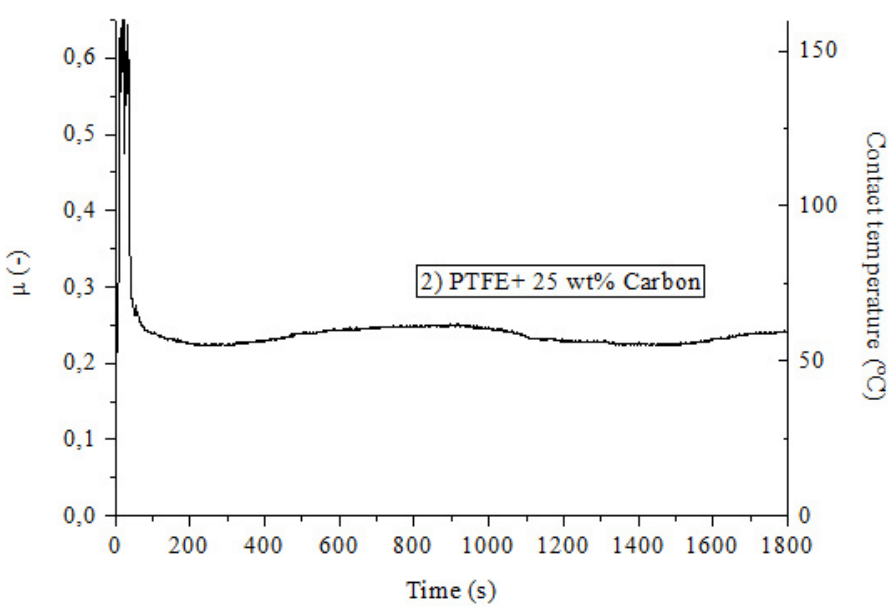

(a)

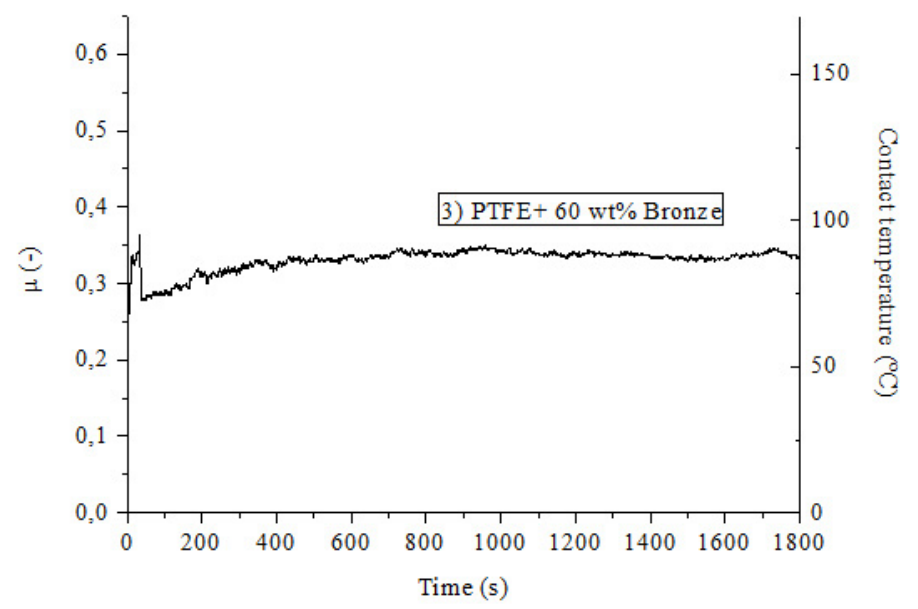

(b)

Figure 5: Coefficient of friction and calculated contact temperature evolution of the PTFE composite samples: (a) carbon particles; (b) bronze particles. 


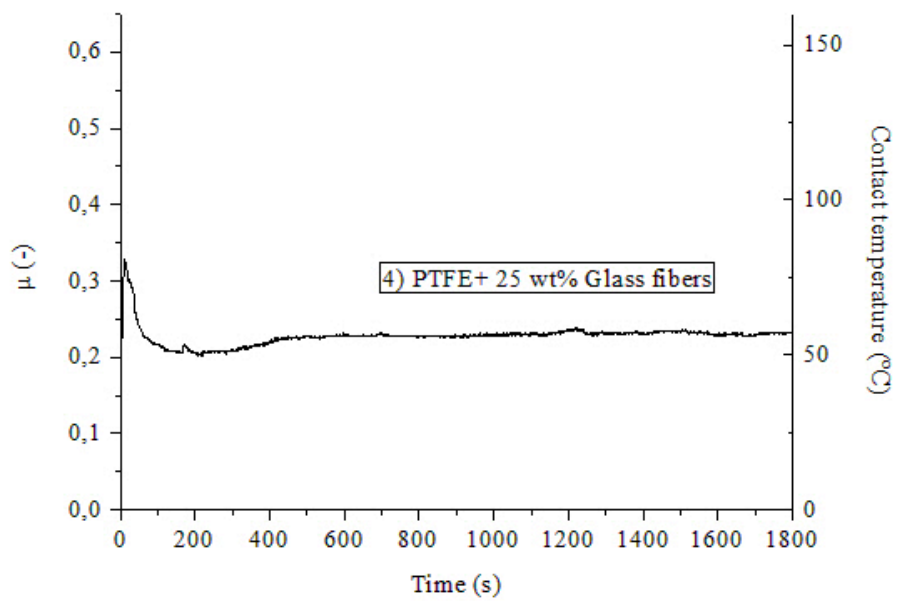

Figure 6: Coefficient of friction and calculated contact temperature evolution of the PTFE glass fiber composite samples.

The energy transformed as a consequence of frictional contact can be dissipated (conversion to heat, vibration, material deformation, new surface creation) or stored into the tribosystem. Therefore, materials exhibiting the same friction coefficient can have different wear rates because the energy is partitioned differently between and within the materials. Both friction coefficient and wear rate are needed for a good understanding of the test. The specific wear energy is a criterion, which takes both into account: it is the ratio of the friction work spent in the interface divided by the mass loss due to the wear:

$$
E_{w}=\frac{E}{\Delta m}=\frac{v \cdot W \cdot \int_{t i}^{t f} \mu(t) \cdot d t}{\Delta m}
$$

where $E$ is the energy dissipated by friction, $v$ is the mean relative sliding velocity, $t_{i}$ the initial time, $t_{f}$ the time at the end of the test, and $\Delta m$ is the total mass loss.

In fig. 7, the frictional energy vs. mass loss is shown in order to identify the best combination of low frictional energy, that is, low friction and less mass loss, which is equivalent to low wear. PTFE composites (2) and (4) meet these criteria and present also the highest values of wear resistance.

For the composites numbered 1, 2 and 3, the small crystals formed at lower temperature are destroyed resulting in flakes separation being less stable: in fact, the calculated contact temperature is around $60-80^{\circ} \mathrm{C}$. PTFE fails along weak intermolecular bonds due to the minor temperature rise which involves slippage of crystalline formations of the bond structure. In other words, the temperature rise is quite relevant as consequence of low thermal conductivity and the energy dissipated when the carbon-to-fluorine bonds, which have high bond dissociation energy, are broken. 


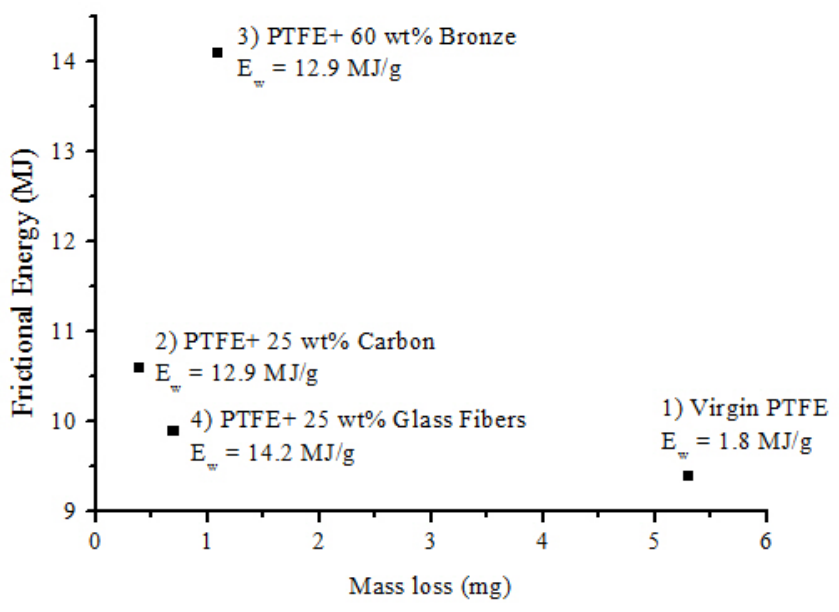

Figure 7: Specific wear energy $E_{w}$.

In figs 8 to 10 , the worn surfaces of PTFE composites can be observed. As expected, the virgin PTFE (fig. 8) shows plucked marks due to the transfer film formation and subsequent adhesion, encouraged by temperature rise at the running in stage. The smallness of the black carbon dispersed particles in the PTFE composite 2 (fig. 9(a)), avoid abrasion of the countersurface due to their selflubricating properties. Bronze hard particles (fig. 9(b)) and glass fibers (fig. 10) act by removing the transfer film and abrading the hard countersurface.

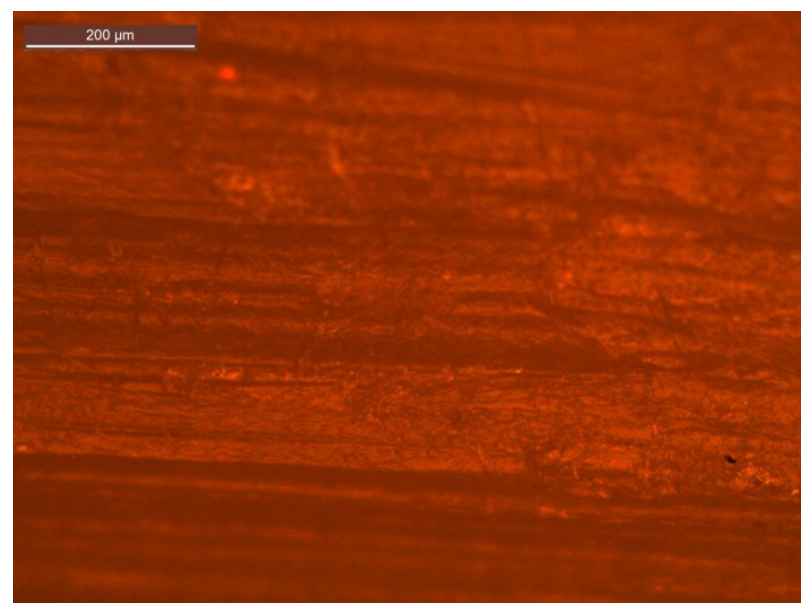

Figure 8: $\quad$ Worn surface of virgin PTFE. 


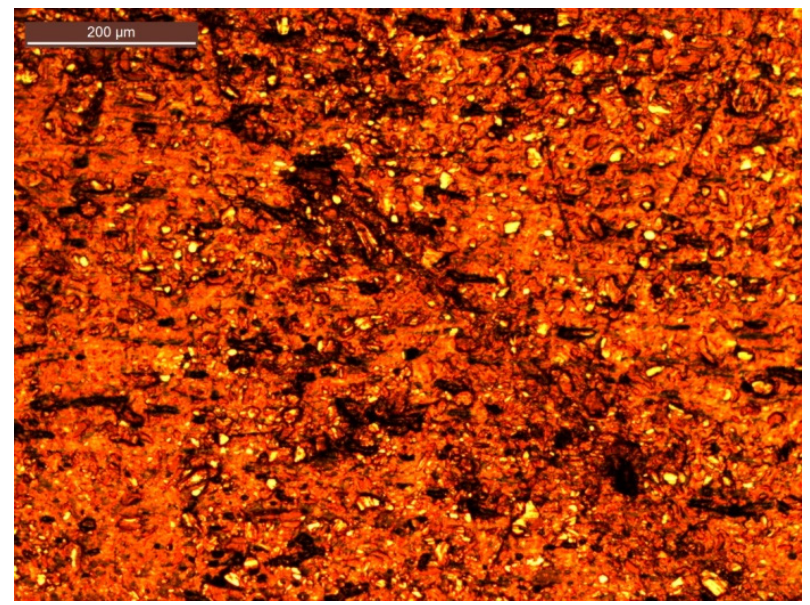

(a)

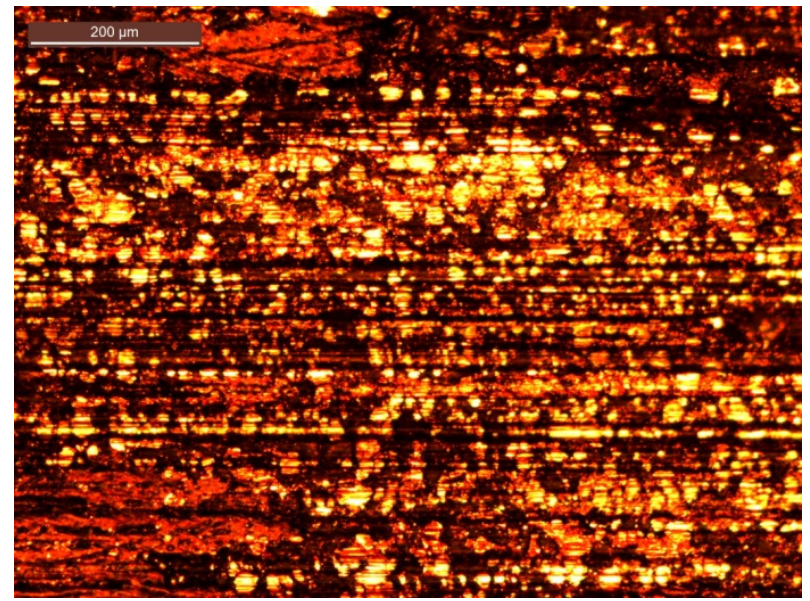

(b)

Figure 9: Worn surfaces of (a) PTFE $+25 \mathrm{wt} \%$ carbon, (b) PTFE+60 wt $\%$ bronze particles. 


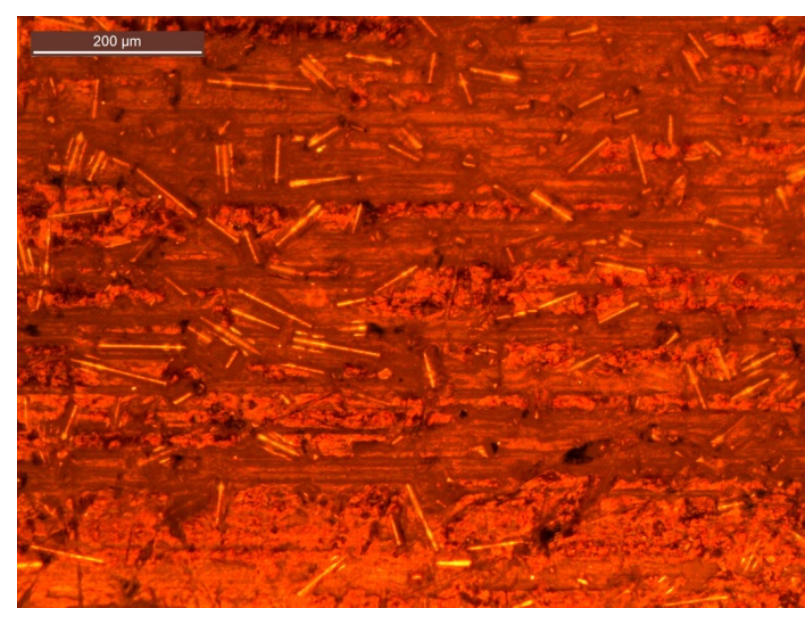

Figure 10: Worn surface of PTFE $+25 \mathrm{wt} \%$ glass fibers.

\section{Conclusions}

The strengthening of the bulk PTFE is well known to be affected by the presence of fillers. On the other hand, tribological characteristics of various PTFE composites are affected by their thermal properties and their structure.

The transition phase at low temperature (from hexagonal to pseudo-hexagonal crystals) allows the formation of a transfer layer, while the crystallinity of the polymer participates in its regeneration.

When polymer composites are considered, their thermal characteristics should be checked with respect to temperature and, furthermore, frictional heating calculations should be carried out when a hard filler is present in the bulk material.

Modelling of the heat flow generated by friction force permits the following considerations:

1. The presence of fillers increases the stability of the structure improving the wear resistance;

2. The increment in thermal conductivity and diffusivity improves wear resistance of the material to adhesion;

3. Bronze particle and glass fiber fillers can reduce the wear mass loss of PTFE under dry conditions significantly but their big size can affect the countersurface and the transfer film;

4. Carbon particles filled PTFE has good combination of thermal characteristics and structure due to the small size of the carbon particles. 


\section{References}

[1] Gong, D., Xue, Q. \& Wang, H., Study of the wear of filled polytetrafluoroethylene. Wear, 134(2), pp. 283-295, 1989.

[2] Conte, M., Fuentes, E., Fernandez, X. \& Igartua, A., Effect of fillers on tribological properties of PTFE composites, Proc. of the 3rd European Conf. on Tribology, Austrian Tribology Society, Wien, 2011.

[3] Aderikha, V.N. \& Shapovalov, V.A., Effect of filler surface properties on structure, mechanical and tribological behavior of PTFE-carbon black composites. Wear, 268, pp. 1455-1464, 2010.

[4] Kalin, M., Influence of flash temperatures on the tribological behaviour in low-speed sliding: a review. Material Science \& Engineering A, 374, pp. 390 397, 2004.

[5] Mao, K., A numerical method for polymer composite gear flash temperature prediction. Wear, 262, pp. 1321-1329, 2007.

[6] Stachowiak, G.W. \& Batchelor, A.W., Engineering Tribology, Elsevier: Amsterdam, pp. 333-345, 2005.

[7] Conte, M., Pinedo, B. \& Igartua, A., Role of crystallinity on wear behaviour of PTFE composites. Wear, 307, pp. 81-86, 2013.

[8] Brown, E.N. \& Dattelbaum, D.M., The role of crystalline phase on fracture and microstructure evolution of polytetrafluoroethylene (PTFE), Polymer, 46, pp. 3056-3068, 2005. 